\title{
COMPUTATIONAL ASSESSMENT OF THE ADME-TOX PROFILES AND HARMFUL EFFECTS OF THE MOST COMMON USED PHTHALATES ON THE HUMAN HEALTH
}

\author{
DANA CRĂCIUNª, DANIELA DASCĂLU ${ }^{\mathrm{b} *}$, ADRIANA ISVORAN ${ }^{\mathrm{b}}$
}

\begin{abstract}
In this study we consider 25 of the most commonly used phthalates and summarize the available data that support their effects on humans. For 15 of the 25 investigated phthalates (60\%) there are no human hazard assessment data, neither from experimental nor from computational studies, which underlines the necessity of their risk assessment. Consequently, we have used various computational tools to predict their ADME-Tox profiles and assess their harmful effects on humans. The outcomes of our study reveal that the investigated phthalates have good bioavailability and skin permeability which are associated with toxicity, especially when they are inhaled. They are able to interact with important molecular targets in the human organism such as membrane receptors, cytochromes, kinases, phosphatases, transcription factors, or transporters. These interactions may conduct to predicted harmful effects of phthalates, such as toxicity and irritations of the respiratory and gastrointestinal tracts, skin and eye irritations, endocrine disruption potential, or non-genotoxic carcinogenicity. The investigated phthalates are not predicted to produce genotoxic carcinogenicity, mutagenicity and cardiotoxicity. Beside the investigated phthalates, the di(2-ethylhexyl) phthalate reflects the highest number of toxic effects, and the ditridecyl phthalate and the diisotrodecyl phthalate illustrate the smallest number of possible toxicological effects, namely skin irritation, non-genotoxic carcinogenicity and endocrine disruption potential.
\end{abstract}

Keywords: phthalates, ADME-Tox, pharmacokinetics, toxicological effects.

a West University of Timisoara, Teacher Training Department, 4 V. Parvan, RO-300223, Timisoara, Romania

b West University of Timisoara, Department of Biology-Chemistry and Advanced Environmental Research Laboratories, 16 Pestalozzi, RO-300115, Timisoara, Romania

*Corresponding author: daniela.dascalu@e-uvt.ro 


\section{INTRODUCTION}

The use of plasticized products has considerably increased in the last decades, exceeding 300 million tons in 2010 [1]. Plastics are synthetic organic polymers of high molecular mass and in order to enhance their physical properties they are often mixed with various additives. Some of these additives, such as phthalates, proved to be of particular concern for the human health. Phthalates are alkyl aryl esters of 1,2-benzenedicarboxylic acid used in the manufacture of plastic as plasticizer agents, i.e. to make hard plastic softer and more flexible. They are also used as solvents in paint and are found in ordinary plastics, common household products, toys, personal care and cosmetics products, medical devices, pharmaceutical products, automobile upholstery and so on. Monitoring data indicated that the general population may be exposed to phthalates via inhalation of outdoor and indoor air and dust, ingestion of food and drinks, by using some pharmaceutical products and by dermal contact with products and/or clothes containing these compounds, and that it is almost impossible to avoid them [2]. Consequently, they can leach in blood and enter the blood circulation [3], with numerous studies confirming the presence of phthalate metabolites in human urine, breast milk and some serum samples. The daily intake of phthalate in humans was estimated at 1.7$52.1 \mu \mathrm{g} / \mathrm{kg} /$ day [4], children exposure being 2-to 4-fold higher than for adults $[5,6]$. Occupational exposure to phthalates may also occur through inhalation and dermal contact at workplaces where they are manufactured or used.

There are numerous in vitro and in vivo studies published concerning the harmful effects of the common phthalates on animals and humans, with most in vivo data being obtained from animal subjects, usually rodents. There are significant differences between animals and humans and, consequently, it is difficult to predict the biological effects of phthalates on humans by using animal subjects [7]. This is the reason why, in this study, we have reviewed only available data that have been obtained on human subjects concerning the biological effects of 25 of the most commonly used phthalates shown in Figure 1.

These data have been extracted from the following sources: specific literature, National Institute for Occupational Safety and Health (NIOSH), Pocket Guide to Chemical Hazards [8], Occupational Safety and Health Administration (OSHA) [9], Toxicology Data Network/ Hazardous Substances Data Bank (TOXNET/HSDB) [10], PubChem [11] databases and BIBRA working groups results [12], respectively. We considered the information available in these databases on the 15th of February 2019.

The target organs of phthalates in humans are: eyes, skin, respiratory system, central and peripheral nervous systems, reproductive system, breast, 
liver, and pancreas $[8,9,11,13]$. The possible effects of phthalates on the human health were also reviewed by Hauser and Calafat (2005) [14], Asghari et al (2015) [15], Katsikantami et al (2016) [16] and Rowdhwal and Chen (2018) [17]. These studies suggest that some of the phthalates produce eyes, nose, and skin irritations conducting to allergies, rhinitis or asthmatic reactions, they are endocrine disrupting compounds producing reproductive and developmental toxicity, cancer, obesity and type 2 diabetes, the harmful effects being usually higher in children $[16,18]$.

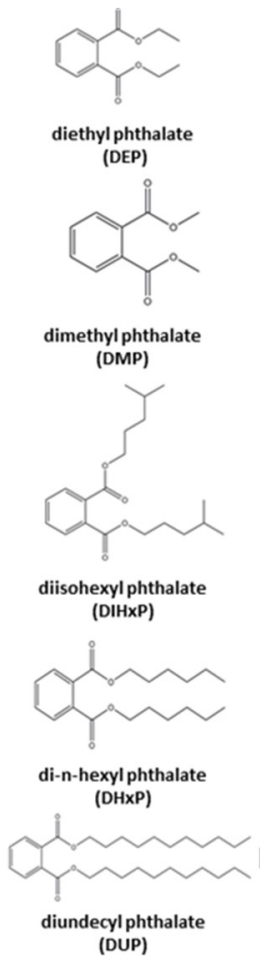

dimethyl phthalate

diisohexyl phthalate

(DHXP)

(DUP)

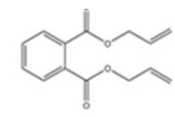

diallyl phthalate

(DAP)

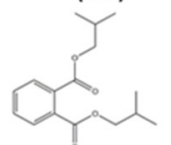

diisobutyl phthalate (DIBP)

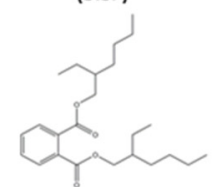

di(2-ethylhexyl) phthalate
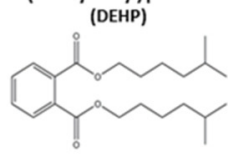

diisoheptyl phthalate (DiHP)

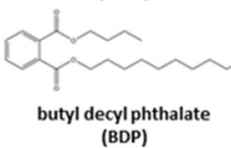

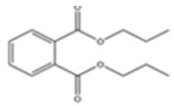

di-n-propyl phthalate (DnPP)

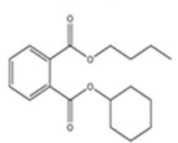

butyl cyclohexyl phthalate (BCP)

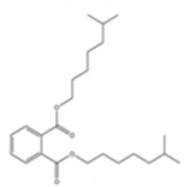

diisooctyl phthalate (DIOP)

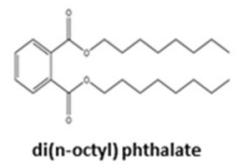
(DnOP)

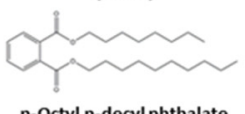
(ODP)

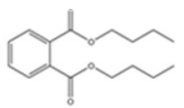

di-n-butyl phthalate (DBP)

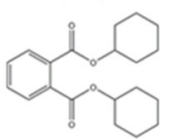

dicyclohexyl phthalate (DCHP)

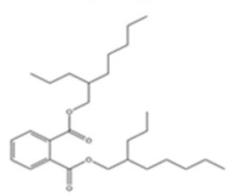

di(2-propylheptyl) phthalate (DPHP)

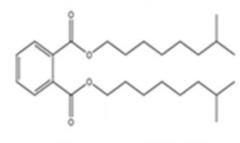

diisononyl phthalate (DINP)

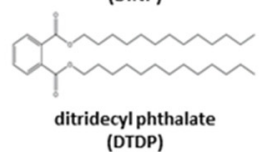
(DTDP)

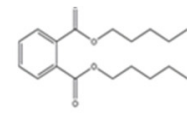

di-n-pentyl phthalate

(DPP)

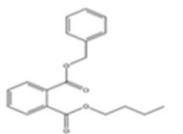

butyl benzyl phthalate (BBP)

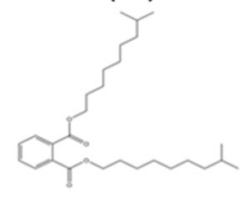

diisodecyl phthalate DIDP)

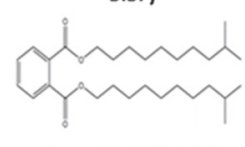

diisoundecyl phthalate (DIUP)

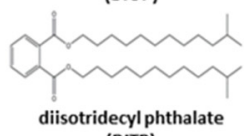
(DITP)

Figure 1. Phthalates considered in this study: formulas, names and abbreviations

Dimethyl phthalate (DMP) may cause irritation of the stomach, dizziness, and unconsciousness [10], the inhalation of its vapors irritates the nose and upper respiratory tract $[8,10,12]$, and it produces skin and eye sensitization and irritation reactions [10, 12]. Diethyl phthalate (DEP) may produce abdominal pain and nausea [11], male reproductive effects [2, 19, 
20, 21], changes in body weight and liver weight, respectively [2]. Prolonged inhalation of DEP causes irritation of the nose, throat and respiratory system, it may produce allergic reactions similar to asthma, and repeated exposure may cause nerve damage [10]. Lopez-Carrillo et al. (2010) suggested a possible connection between exposure to DEP through cosmetics and personal care products and an increased risk of breast cancer [22]. Di-n-butyl phthalate (DBP) may cause abdominal pain, nausea, diarrhea, vomiting, dizziness and headache [10,12], may produce adverse respiratory outcomes [23], endometriosis, and has an anti-androgenic action [10]. DBP provokes skin and eye irritations, dermatitis [10], and prenatal exposure to DBP may cause adverse effects on the neurodevelopment and the behavior of young children [24]. Diisobutyl phthalate (DIBP) may cause male reproductive tract development problems [2]. Di-n-pentyl phthalate (DPP) may produce central nervous system depression and dermal irritations [10]. Butyl benzyl phthalate (BBP) is associated with rhinitis and eczema in children [25], may produce skin irritations [12] and endometriosis [10], and its presence is associated to airway inflammation in children [10]. Di(2-ethylhexyl) phthalate (DEHP) may cause irritation of eyes and mucous membranes, mild gastric disturbances and moderate diarrhea, it affects the reproductive system and the gestational age for newborns, and conducts to a decrease in the hemoglobin level [10]. Matsumoto et al. (2008) observed an association between higher DEHP serum levels and a shorter duration of pregnancy [26]. Literature also shows a positive correlation between phthalate metabolites in urine and symptoms of attention-deficit/hyperactivity disorder ADHD among school-age children [10]. Maternal exposure to DEHP may affect sex steroid hormones' status in the fetal and newborn stages [27, 28]. Prenatal and postnatal exposure was associated with the occurrence of asthma in children, particularly in boys [29], and has adverse effects on the neurodevelopment and the behavior of young children [23]. DEHP may produce changes to the male reproductive tract $[2,30,31]$ and adversely affect the liver and thyroid $[2,10]$. DEHP can cause endometriosis in reproductive-age women [32,33], opacification of the lungs in pre-term infants [10], and is associated with allergic symptoms in children [34]. Di(n-octyl) phthalate (DnOP) may produce endometriosis, asthmatic reaction and skin irritation [10]. Diisodecyl phthalate (DIDP) may produce asthma and have an anti-androgenic action, and may affect the male reproductive system [10]. Two cases of allergic dermatitis as a result of occupational exposure to DIDP have been registered [10].

No human studies were available for $15(60 \%)$ of the 25 phthalates under investigation. In humans, phthalates are metabolized to their monoesters that may also reflect different degrees of toxicity, but we underline that we have not considered these metabolites in our study. 
Some studies suggest that the quantity and quality of the available data are not consistent [2,35], and that there is no clear indication of the harmful effects of every phthalate in humans [2, 14, 36-38]. Also, as humans are continuously exposed to various combinations of chemical compounds, and phthalates are only one component of this mixture, some of the findings could not be exclusively associated to phthalates and it is difficult to obtain objective information concerning their exposure and epidemiologic data.

The concerns regarding the effects of phthalate exposure has led the U.S. Food and Drug Administration (FDA) and the European Medicines Agency (EMA) to establish guidelines on patient exposure to some phthalates if they are present in pharmaceutical products. Nevertheless, human contamination with phthalate may occur from a number of different other sources.

In our opinion, there are insufficient or missing data concerning the health risks of some phthalates, and further research on evaluating their effects in humans is needed. As it is not a simple task and humans are not volunteers for such experiments, computational analysis may be used to predict the biological effects of phthalates in humans. These predictions may be further investigated by experimental techniques. There is a great variety of computational tools available for the assessment of the biological effects of chemicals, most of them being designed for studying drugs, and the continuous development of such tools can be observed. A comprehensive overview can be found in the review of Raies and Bajic (2016) [39]. Furthermore, the specific literature shows promising results concerning the computational assessment of the toxicity of different chemicals other than drugs [39-47], reflecting the actuality of these research tools. Moreover, a recent study found that the existing models available for predicting various toxicity endpoints of drugs could be successfully used for industrial chemicals [48]. These tools also limit the amount of animal testing and the environmental effects, and reduce the costs of research and development activities. Consequently, in this study, we use various computational approaches for predicting the absorption, distribution, metabolism, excretion and toxicity (ADME-Tox) profiles, the pharmacokinetics, the molecular targets, the biological activity spectra and toxicological endpoints for the most commonly used phthalates (Figure 1). The predictions are further compared to each other and to the known effects of each considered phthalate in humans (when available). The correlations between computational results and in vivo experimental data are discussed.

\section{RESULTS AND DISCUSSIONS}

The use of the FAFDrugs 4 tool to produce the ADME-Tox profiles of the phthalates considered in this study leads to the results presented in Table 1 
with two filters corresponding respectively to the ingestion and inhalation as routes of exposure. Compounds not fulfilling the selected filters and/or presenting a high-risk structural alert do not reveal bioavailability and/or reflect high toxicity, and are considered not to be used by humans, their status being set to "rejected" in Table 1 [49].

The results obtained using the FAFDrugs4 computational tool reveal that all considered phthalates expose good oral bioavailability and at least reduced toxicity, regardless of the exposure modality. None of the phthalates pass the Pfizer $3 / 75$ rule for toxicity and the high molecular weight phthalates also do not respect the GSK 4/400 rule.

Table 1. ADME-Tox profiles of phthalates: white boxes reveal that the rules are respected and grey boxes reflect that the rules are broken. Accepted compounds are those reflecting a good oral bioavailability and low toxicity when used by humans. Rejected compounds are those revealing medium to high toxicity.

\begin{tabular}{|c|c|c|c|c|c|c|c|}
\hline \multirow[t]{2}{*}{ Phthalate } & \multirow{2}{*}{$\begin{array}{l}\text { route of } \\
\text { exposure }\end{array}$} & \multicolumn{3}{|c|}{ Oral Bioavailability } & \multicolumn{2}{|c|}{ Safety profile } & \multirow[t]{2}{*}{ status } \\
\hline & & $\begin{array}{l}\text { R05 } \\
\text { violations }\end{array}$ & $\begin{array}{l}\text { Veber } \\
\text { Rule }\end{array}$ & $\begin{array}{l}\text { Egan } \\
\text { Rule }\end{array}$ & $\begin{array}{l}\text { GSK } \\
\text { Rule }\end{array}$ & $\begin{array}{l}\text { Pfizer } \\
\text { Rule }\end{array}$ & \\
\hline \multirow[t]{2}{*}{ DMP } & ingestion & 0 & Good & good & good & warning & accepted \\
\hline & inhalation & 0 & Good & good & good & warning & rejected \\
\hline \multirow[t]{2}{*}{ DEP } & ingestion & 0 & Good & good & good & warning & accepted \\
\hline & inhalation & 0 & Good & good & good & warning & rejected \\
\hline \multirow[t]{2}{*}{ DAP } & ingestion & 0 & Good & good & good & warning & accepted \\
\hline & inhalation & 0 & Good & good & good & warning & rejected \\
\hline \multirow{2}{*}{ DnPP } & ingestion & 0 & Good & good & good & bad & accepted \\
\hline & inhalation & 0 & Good & good & good & bad & rejected \\
\hline \multirow[t]{2}{*}{ DBP } & ingestion & 0 & Good & good & good & bad & accepted \\
\hline & inhalation & 0 & Good & good & good & bad & rejected \\
\hline \multirow[t]{2}{*}{ DIBP } & ingestion & 0 & Good & good & good & bad & accepted \\
\hline & inhalation & 0 & Good & good & good & bad & rejected \\
\hline \multirow[t]{2}{*}{$\mathrm{BCP}$} & ingestion & 0 & Good & good & good & bad & accepted \\
\hline & inhalation & 0 & Good & good & good & bad & rejected \\
\hline \multirow[t]{2}{*}{ DPP } & ingestion & 1 & Good & good & good & bad & rejected \\
\hline & inhalation & 1 & Good & good & good & bad & rejected \\
\hline \multirow[t]{2}{*}{ BBP } & ingestion & 0 & Good & good & good & bad & rejected \\
\hline & inhalation & 0 & Good & good & good & bad & rejected \\
\hline \multirow[t]{2}{*}{$\mathrm{DCHP}$} & ingestion & 1 & Good & good & good & bad & accepted \\
\hline & inhalation & 1 & Good & good & good & bad & rejected \\
\hline \multirow[t]{2}{*}{$\mathrm{DHxP}$} & ingestion & 1 & Good & good & good & bad & rejected \\
\hline & inhalation & 1 & Good & good & good & bad & rejected \\
\hline
\end{tabular}




\begin{tabular}{|c|c|c|c|c|c|c|c|}
\hline \multirow[t]{2}{*}{ DIHXP } & ingestion & 1 & Good & good & good & bad & accepted \\
\hline & inhalation & 1 & Good & good & good & warning & rejected \\
\hline \multirow[t]{2}{*}{$\mathrm{DiHP}$} & ingestion & 1 & Good & good & good & bad & rejected \\
\hline & inhalation & 1 & Good & good & good & bad & rejected \\
\hline \multirow[t]{2}{*}{ BDP } & ingestion & 1 & Good & good & good & bad & rejected \\
\hline & inhalation & 1 & Good & good & good & bad & rejected \\
\hline \multirow[t]{2}{*}{ DEHP } & ingestion & 1 & Good & good & good & bad & rejected \\
\hline & inhalation & 1 & Good & good & good & bad & rejected \\
\hline \multirow[t]{2}{*}{ DnOP } & ingestion & 1 & Good & good & good & bad & rejected \\
\hline & inhalation & 1 & Good & good & good & bad & rejected \\
\hline \multirow[t]{2}{*}{ DIOP } & ingestion & 1 & Good & good & good & bad & rejected \\
\hline & inhalation & 1 & Good & good & good & bad & rejected \\
\hline \multirow[t]{2}{*}{ ODP } & ingestion & 1 & Good & good & bad & bad & rejected \\
\hline & inhalation & 1 & Good & good & bad & bad & rejected \\
\hline \multirow[t]{2}{*}{ DINP } & ingestion & 1 & Good & good & bad & bad & rejected \\
\hline & inhalation & 1 & Good & good & bad & bad & rejected \\
\hline \multirow[t]{2}{*}{ DPHP } & ingestion & 1 & Good & good & bad & bad & rejected \\
\hline & inhalation & 1 & Good & good & bad & bad & rejected \\
\hline \multirow[t]{2}{*}{ DIDP } & ingestion & 1 & Good & good & bad & bad & rejected \\
\hline & inhalation & 1 & Good & good & bad & bad & rejected \\
\hline \multirow[t]{2}{*}{ DIUP } & ingestion & 1 & Good & good & bad & bad & rejected \\
\hline & inhalation & 1 & Good & good & bad & bad & rejected \\
\hline \multirow[t]{2}{*}{ DUP } & ingestion & 1 & Good & good & bad & bad & rejected \\
\hline & inhalation & 1 & Good & good & bad & bad & rejected \\
\hline \multirow[t]{2}{*}{ DTDP } & ingestion & 2 & Good & good & bad & bad & rejected \\
\hline & inhalation & 2 & Good & good & bad & bad & rejected \\
\hline \multirow[t]{2}{*}{ DITP } & ingestion & 2 & Good & good & bad & bad & rejected \\
\hline & inhalation & 2 & Good & good & bad & bad & rejected \\
\hline
\end{tabular}

Most of the phthalates have the status set to rejected, especially when inhaled. The most frequent reasons for rejecting these phthalates are: too many rotatable bonds, high values of partition coefficient, too few hydrogen bonds donors and acceptors, and the presence of consecutive alkyl chains as high risk structural alerts. The good oral bioavailability is also predicted when using the admetSAR tool [50] (see Table 2 below). This tool has been also used to predict the pharmacokinetic profiles of the phthalates, presented in Table 2 together with predictions concerning the inhibition of the human CYPs that were obtained using the SwissADME computational tool [51].

The results presented in Table 2 reveal that, usually, low molecular weight phthalates expose high gastrointestinal absorption and blood-brainbarrier permeability. The predictions concerning the high gastrointestinal absorption and the blood-brain barrier permeability of the low molecular 
weight phthalates are in accordance with the observed effects on humans for acute exposure: gastrointestinal disorders (DMP, DEP, DBP, DEHP) [10] and central nervous system disorders (DEP, DBP, DPP, DEHP) [10, 23]. These outcomes reveal that the investigated phthalates are not considered to be inhibitors of the renal organic cationic transporter. Some of the investigated phthalates are predicted to be able to inhibit the human CYPs, with smaller molecular weight phthalates usually affecting CYP1A2 and CYP2C9 and higher molecular weight phthalates affecting CYP3A4. These interactions are assessed further by molecular docking.

As dermal contact is one of the ways of exposure to phthalates, we have computed their skin permeation coefficients using the SwissADME tool [51] and predicted their skin sensitization potentials using the PredSkin tool $[52,53]$. The values of the skin permeation coefficients are revealed in Figure 2. A larger negative value of logKp corresponds to a less skin permeant compound. It is known that $\log \mathrm{Kp}=-4.96$ for diclofenac, an anti-inflammatory drug with a good skin permeability [51].

Table 2. SwissADME and admetSAR predictions concerning the pharmacokinetic profile of phthalates: BBB+- permeation of the blood-brain barrier, $\mathrm{Gl}+-$ gastrointestinal absorption, P-gp - glycoprotein P, ROCT - renal organic cationic transporter, CYPs - human cytochromes.

\begin{tabular}{|l|l|l|l|l|l|l|l|l|l|}
\hline \multirow{2}{*}{ Phthalate } & \multicolumn{4}{|c|}{ Probability of } & \multicolumn{3}{|c|}{$\begin{array}{c}\text { SwissADME predictions concerning } \\
\text { the inhibition of the human CYPs }\end{array}$} \\
\cline { 2 - 11 } & BBB & GI & $\begin{array}{c}\text { P-gp } \\
\text { substrate }\end{array}$ & $\begin{array}{c}\text { ROCT } \\
\text { non- } \\
\text { inhibitor }\end{array}$ & $\begin{array}{c}\text { CYP } \\
\text { 1A2 }\end{array}$ & $\begin{array}{l}\text { CYP } \\
\text { 2C19 }\end{array}$ & $\begin{array}{l}\text { CYP } \\
\text { 2C9 }\end{array}$ & $\begin{array}{l}\text { CYP } \\
\text { 2D6 }\end{array}$ & $\begin{array}{l}\text { CYP } \\
\text { 3A4 }\end{array}$ \\
\hline DMP & 0.958 & 0.960 & -0.736 & 0.917 & Yes & No & No & No & No \\
\hline DEP & 0.926 & 0.975 & -0.691 & 0.889 & Yes & No & No & No & No \\
\hline DAP & -0.957 & 0.920 & -0.724 & 0.837 & Yes & Yes & No & No & No \\
\hline DnPP & 0.933 & 0.976 & -0.618 & 0.868 & Yes & Yes & No & No & No \\
\hline DBP & 0.939 & 0.979 & -0.547 & 0.849 & Yes & Yes & No & No & No \\
\hline DIBP & 0.930 & 0.974 & -0.646 & 0.891 & Yes & Yes & No & No & No \\
\hline BCP & -0.902 & 0.994 & 0.544 & 0.730 & Yes & Yes & Yes & No & No \\
\hline DPP & 0.955 & 0.972 & 0.522 & 0.847 & Yes & No & Yes & No & No \\
\hline BBP & 0.934 & 0.986 & -0.677 & 0.785 & Yes & Yes & No & Yes & No \\
\hline DCHP & -0.943 & 0.972 & 0.515 & 0.768 & No & No & Yes & No & No \\
\hline DHxP & 0.945 & 0.977 & 0.500 & 0.826 & Yes & No & Yes & No & No \\
\hline DIHxP & 0.945 & 0.943 & 0.558 & 0.832 & No & No & No & No & No \\
\hline DiHP & 0.940 & 0.946 & 0.509 & 0.839 & No & No & No & No & No \\
\hline BDP & 0.945 & 0.979 & 0.500 & 0.844 & Yes & No & Yes & No & Yes \\
\hline DEHP & 0.938 & 0.977 & 0.500 & 0.826 & No & No & Yes & No & Yes \\
\hline DnOP & -0.945 & 0.954 & -0.511 & 0.818 & No & No & No & No & Yes \\
\hline DIOP & 0.927 & 0.954 & -0.511 & 0.818 & No & No & No & No & No \\
\hline ODP & 0.938 & 0.979 & 0.500 & 0.844 & No & No & No & No & Yes \\
\hline
\end{tabular}




\begin{tabular}{|l|l|l|l|l|l|l|l|l|l|}
\hline DINP & 0.927 & 0.954 & -0.511 & 0.818 & No & No & No & No & Yes \\
\hline DPHP & 0.927 & 0.954 & -0.511 & 0.818 & No & No & Yes & No & Yes \\
\hline DIDP & 0.945 & 0.977 & 0.500 & 0.826 & No & No & No & No & No \\
\hline DIUP & 0.945 & 0.977 & 0.500 & 0.826 & No & No & No & Yes & No \\
\hline DUP & 0.945 & 0.977 & 0.500 & 0.826 & No & No & No & Yes & No \\
\hline DTDP & 0.945 & 0.977 & 0.500 & 0.826 & No & No & No & No & No \\
\hline DITP & 0.927 & 0.954 & 0.511 & 0.818 & No & No & No & No & No \\
\hline
\end{tabular}

Figure 2 illustrates that most of the investigated phthalates, especially those with a higher molecular weight, are predicted as having good skin permeability. This outcome is in good correlation with the Pred-Skin predictions (Figure 3), revealing that numerous of the investigated phthalates have a skin sensitization potentiala complex immunological disease having an essential impact on the quality of life and on the working abilities of people professionally exposed to them. Furthermore, these predictions correspond to known data reflecting the skin irritation potential of DEP, DAP, DBP, DPP, BBP, DNOP, DIDP [10].

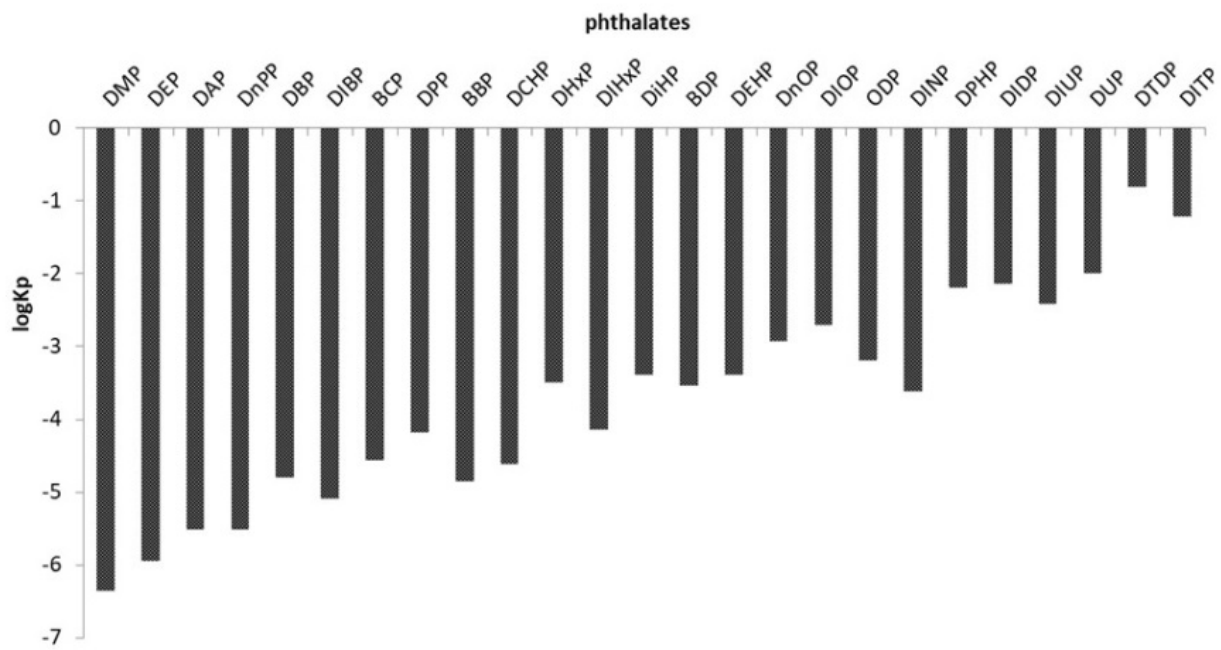

Figure 2. Skin permeation coefficients (logarithmic values) for the investigated phthalates.

The predictions concerning the possible endocrine disruption potential of investigated phthalates, computed using the ENDOCRINE DISRUPTOME tool [54], are shown in Table 3, and reflect that all investigated phthalates could have a low antagonistic effect on the androgen receptor. Antiandrogenic effects have been reported for DBP and DIDP in literature [10]. Other nuclear receptors affected by a large number of the studied phthalates are the glucocorticoid receptor (GR) and the thyroid receptors $\alpha(T R \alpha)$ and $\beta$ 
(TR $\beta$ ). It is not surprising that the androgen and glucocorticoid receptors are quite similarly affected by the studied phthalates, as it is already known that the nuclear hormone receptors share highly conserved ligand binding domains [55]. DCHP is considered to have moderate agonistic and antagonistic effects on both androgen and estrogen receptors and it may also act on the glucocorticoid receptor and on the thyroid receptors. DCHP and DIOP are predicted to have the highest endocrine disruption potentials as they may affect numerous nuclear receptors.

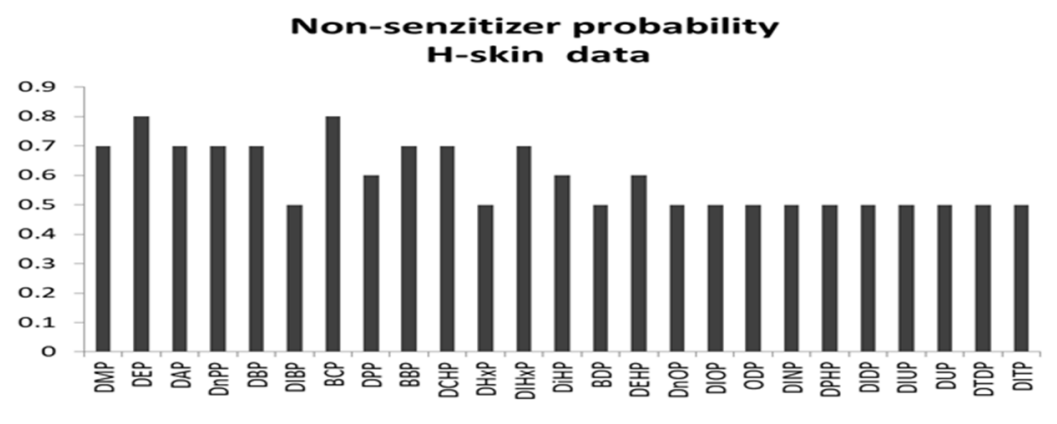

\section{Senzitizer probability} h-CLAT test

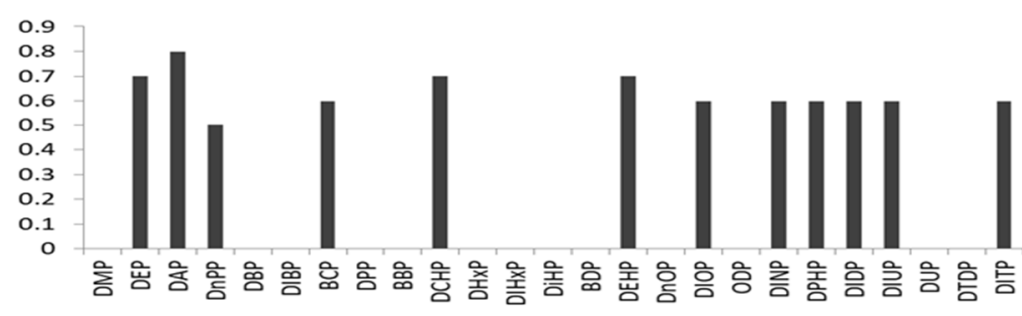

Senzitizer probability KerationSensTM test

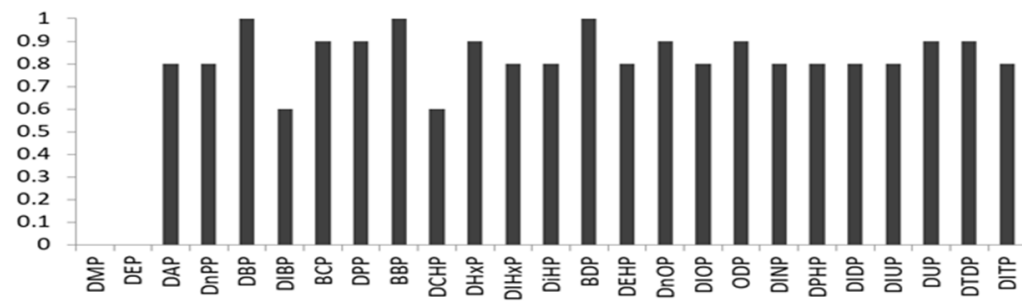

Figure 3. Predictions concerning skin sensitization potentials of the investigated phthalates. White boxes reflect a non-sensitizer potential, light grey boxes reflect a moderate sensitizer potential and dark grey boxes reflect a high sensitizer potential. There are predictions based on human data (H-skin), h-CLAT (human

Cell Line Activation Test) and KeratinoSensTM tests. 
The Pred-hERG [56] computational tool has been used to obtain information concerning the ability of the investigated phthalates to inhibit the hERGK+ channels, the results being presented in Figure 4 . Both binary and multiclass prediction models predict a non-inhibitory potential of the hERGK ${ }^{+}$ channel. Furthermore, specific literature does not mention cardiotoxicity among the toxicological endpoint of these phthalates.

The use of the Toxtree [57] computational facility to assess the harmful effects of the investigated phthalates illustrates that they belong to the chemical class of low toxicity (except DAP which reflects an intermediate toxicity), they do not reveal a genotoxic carcinogenicity and mutagenicity, and that all of the investigated phthalates present a structural alert (phthalate diesters as peroxisome proliferators) for non-genotoxic carcinogenicity (Table 4).
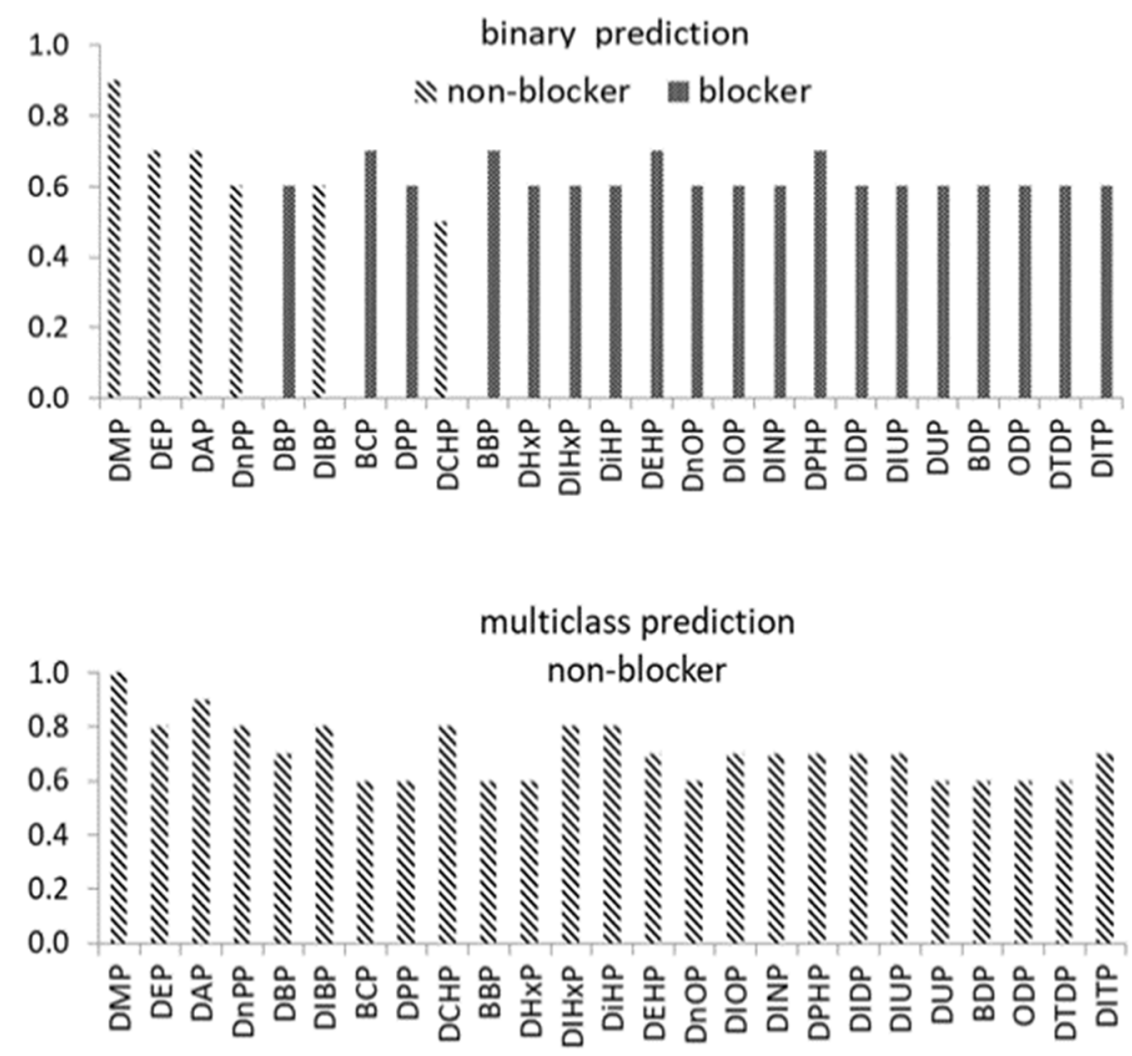

Figure 4. Reliability of the predictions concerning the inhibition of hERGK+ channel 


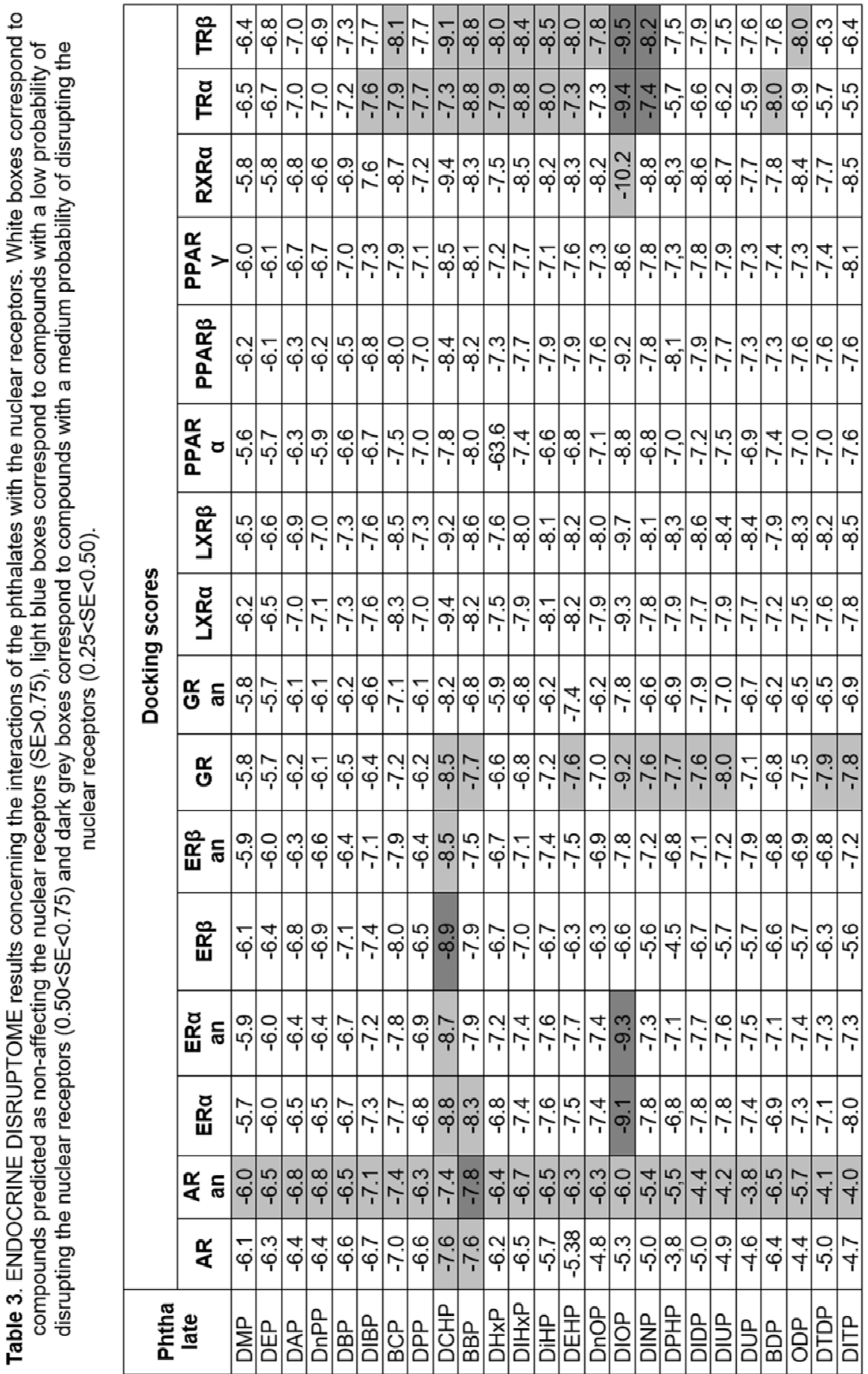


Table 4. Predictions obtained using the Toxtree software concerning the harmful effects of the investigated phthalates.

\begin{tabular}{|c|c|c|c|c|c|c|}
\hline $\begin{array}{c}\text { Phthalate/ } \\
\text { predicted } \\
\text { biological } \\
\text { effects }\end{array}$ & $\begin{array}{c}\text { Skin irritation } \\
\text { and } \\
\text { corrosion }\end{array}$ & $\begin{array}{c}\text { Eyes } \\
\text { irritation }\end{array}$ & $\begin{array}{c}\text { Genotoxic } \\
\text { Irritation }\end{array}$ & No & No & Non-genotoxic \\
carcinogenicity & Mutagenicity & (Ames test) & $\begin{array}{c}\text { Toxicity } \\
\text { class }\end{array}$ \\
\hline DMP & Irritation & No & No & Yes & No & Low \\
\hline DEP & Irritation & Low \\
\hline DAP & Irritation & No & No & Yes & No & Intermediate \\
\hline DnPP & Irritation & No & No & Yes & No & Low \\
\hline DBP & Irritation & No & No & Yes & No & Low \\
\hline DIBP & Irritation & No & No & Yes & No & Low \\
\hline BCP & Irritation & No & No & Yes & No & Low \\
\hline DPP & Irritation & No & No & Yes & No & Low \\
\hline BBP & Irritation & No & No & Yes & No & Low \\
\hline DCHP & Irritation & No & No & Yes & No & Low \\
\hline DHxP & Irritation & No & No & Yes & No & Low \\
\hline DIHxP & Irritation & No & No & Yes & No & Low \\
\hline DiHP & Not corrosive & No & No & Yes & No & Low \\
\hline BDP & Not corrosive & No & No & Yes & No & Low \\
\hline DEHP & Not corrosive & No & No & Yes & No & Low \\
\hline DnOP & Not corrosive & No & No & Yes & No & Low \\
\hline DIOP & Not corrosive & No & No & Yes & No & Low \\
\hline ODP & Not corrosive & No & No & Yes & No & Low \\
\hline DINP & Not corrosive & No & No & Yes & No & Low \\
\hline DPHP & Not corrosive & No & No & Yes & No & Low \\
\hline DIDP & Not corrosive & No & No & Yes & No & Low \\
\hline DIUP & Not corrosive & No & No & Yes & No & Low \\
\hline DUP & Not corrosive & No & No & Yes & No & Low \\
\hline DTDP & Not corrosive & No & No & Yes & No & Low \\
\hline DITP & Not corrosive & No & No & Yes & No & Low \\
\hline
\end{tabular}

The assessment of the interactions of the phthalates with CYPs has been performed using the SwissDock software [58]. The illustrations concerning the results furnished by this computational tool are revealed in Figure 5 and all the free energies of the predicted interactions are exposed in Figure 6. Figure 5a illustrates the predicted binding modes (BMs) for the interaction of DHxP (presented as dark grey sticks) with CYP1A2 (presented as a light grey cartoon), none of them corresponding to the active site, the location of which is shown by the presence of the prosthetic group hem (dim grey sticks) and the inhibitor alpha-naphthoflavone (black sticks). Figure $5 \mathrm{~b}$ reveals the $\mathrm{BM}$ with the highest free energy for the interaction of DEHP (shown in dark grey sticks) with the active site of CY3A4. The protein is shown as a light grey cartoon, the prosthetic group hem is revealed as a light grey wire, while 
the inhibitor tert-butyl \{6-oxo-6-[(pyridin-3-ylmethyl)amino]hexyl\}carbamate is shown as black sticks. Figure $5 \mathrm{~b}$ illustrates that the binding mode of DEHP corresponds to the position of the inhibitor tert-butyl \{6-oxo-6-[(pyridin-3ylmethyl)amino]hexyl\}carbamate and underlines the inhibitory potential of DEHP on CYP3A4.

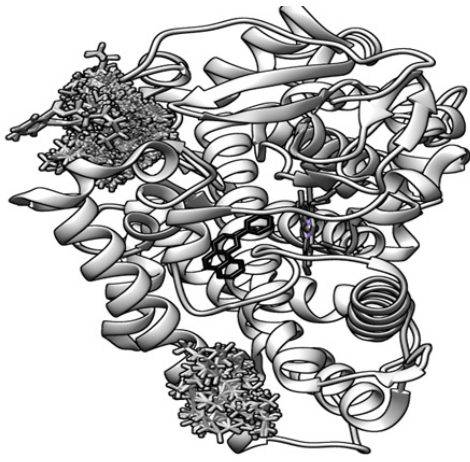

a

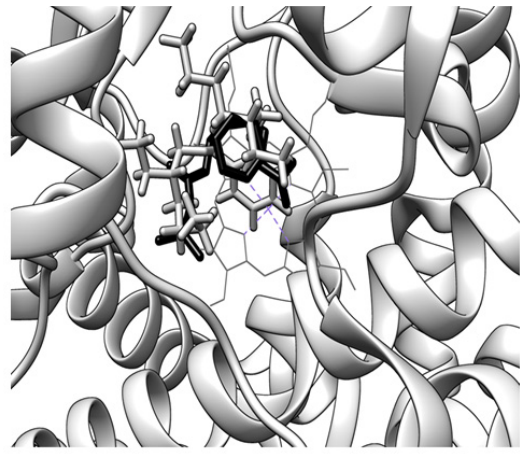

b

Figure 5. (a) Illustration of the binding modes of di-n-hexyl phthalate (dark grey sticks) to the CYP1A2 (light grey cartoon). The hem group is illustrated with dim grey sticks and the inhibitor alpha-naphthoflavone that is present in the crystallographic structure of the CYP1A2 is revealed using black sticks. (b) Illustration of the binding of di(2-ethylhexyl) phthalate (dark grey sticks) to the active site of CY3A4. The protein is shown as a light grey cartoon, the prosthetic group hem is revealed as a light grey wire and the inhibitor tert-butyl \{6-oxo-6-

[(pyridin-3-ylmethyl)amino]hexyl\}carbamate is shown using black sticks

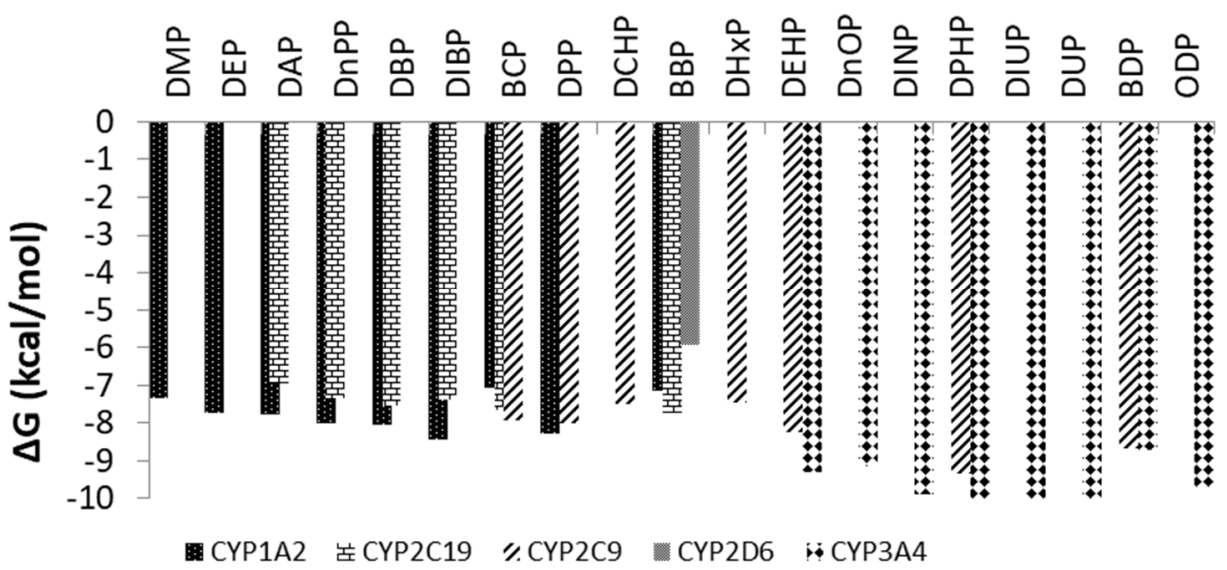

Figure 6. Computed free energies for the interactions of the phthalates with human cytochromes involved in the metabolism of xenobiotics 
Figure 6 emphasizes that almost all predictions made by the SwissADME tool concerning the inhibition of cytochromes known to be involved in the metabolism of xenobiotics (including drugs) are confirmed by the molecular docking study. DHxP and BDP are predicted to bind to CYP1A2, although there are no binding modes corresponding to the active site of the enzyme (as it is illustrated in the Figure 5a for the interaction of DHxP with CYP1A2). These predictions do not exclude the inhibitory potential of DHxP and BDP on CYP1A2, as these interactions may have allosteric effects on the biological activity of the enzyme. Experimental data confirm the interaction of DEHP with CYP2C9, this cytochrome being responsible for the metabolism of DEHP [59]. The inhibitory potential of phthalates on the cytochromes is important to be considered because these enzymes are involved in the metabolism of numerous endogenous and exogenous compounds (drugs and other xenobiotics).

\section{CONCLUSIONS}

Considering the high development rate of the industries of food and drinks, furnishing and paint, cosmetics, hygiene, or of pharmaceutical and medical products, the potential exposure and accumulation of phthalates in the human body is increased which emphasizes the necessity of assessing the risks of these chemicals. A special attention is needed for people who are professionally exposed to a higher amount of phthalates. Within this study we have used various computational tools to investigate the toxicity of 25 of the most commonly used phthalates for humans. To the best of our knowledge, for 12 of the 25 investigated phthalates (48\%), this is the first study providing data concerning their effects on humans. The predictions made by the various computational tools used in this study are in good agreement with each other and also with published data concerning the effects on human health observed for some of the investigated phthalates which increases the value of predictions concerning the toxicological effects for the phthalates that were not investigated. The obtained results reveal that phthalates are a class of compounds reflecting oral bioavailability, skin penetration and toxicity. When they are inhaled, their toxicity is increased. The investigated phthalates are predicted to be able to interact with important molecular targets in the human organism (membrane receptors, kinases, phosphatases, transcription factors and transporters) and these interactions may disrupt the physiological activity of the targets, conducting to predicted harmful effects such as skin irritation, antagonistic effect on some nuclear receptors, non-genotoxic carcinogenicity, or inhibition of cytochromes involved in the metabolism of 
xenobiotics (especially CYP1A2 and CYp3A4). The investigated phthalates do not reveal a genotoxic carcinogenicity and mutagenicity. Ditridecyl phthalate and diisotrodecyl phthalate illustrate the smallest number of possible toxicological effects, namely skin irritation, non-genotoxic carcinogenicity and endocrine disruption potential. Our study reveals that lower molecular weight phthalates are predicted to be able to conduct to almost all investigated toxicological endpoints. Thus, we consider these phthalates to be the most dangerous ones and recommend that their use be avoided. Finally, we are of the opinion that this study highlights the necessity of further complex experimental investigations concerning the harmful effects of phthalates on humans.

\section{EXPERIMENTAL SECTION}

Our study concerns the application of the following computational tools to envisage the possible biological effects on humans of the most commonly used phthalates: (i) the FAFDrugs4 tool [49] for producing the ADME-Tox profiles; (ii) admetSAR [50] and SwissADME [51] for envisaging pharmacokinetic profiles [50]; (iii) Pred-Skin for predicting skin sensitization [52, 53]; (iv) ENDOCRINE DISRUPTOME for predicting endocrine disruption potential [54]; (v) Pred-hERG for predicting the inhibitory potential against hERGK+ channel [56]; (v) ToxTree for predicting toxicity class, carcinogenicity and mutagenicity [57]; and (vii) SwissDock [58] to assess the interactions of the cytochromes P450 (CYP) involved in xenobiotics metabolism (CYP1A2, CYP2C9, CYP2C19, CYP3A4, CYP2D6) with phthalates.

Absorption, Distribution, Metabolism, Excretion and Toxicity (ADMETox) profiles are commonly used to predict disposition of drugs within the human organism in the initial stage of their design. There are several in silico approaches used to predict the ADME-Tox profiles. These approaches are centered on a set of rules based on the physicochemical properties of the chemical compound that are used to predict its bioavailability and safety. The physicochemical properties are computed by the computational tools enumerated in the previous paragraph starting from the Simplified MolecularInput Line-Entry System (SMILES) formulae of the investigated phthalates. The SMILES formulae are extracted from the ZINC [60] and PubChem [11] databases, respectively. The bioavailability designates the absorbed part of the ingested amount of a chemical compound. In order to predict the bioavailability, the physicochemical properties reflecting the hydrophilicity and solubility of a chemical compound are important and the following rules are commonly used: the rule-of-5 violations (RO5, also known as the Lipinski's rule) [61], Egan's Rule [62], and Veber's Rule [63]. The compounds 
with more than one violation of the RO5 rule reflect poor absorption. The safety profile illustrates the adverse effects of a chemical compound and is based on a few other common rules, such as the GlaxoSmithKline rule [64] and the Pfizer $3 / 75$ rule [65].

The human exposure to phthalates can take place by ingestion, inhalation, dermally and/or eye contact, or by intravenous injection through medical devices. When producing the ADME-Tox profiles for phthalates using the FAFDrugs 4 computational tool, the routes of exposure through ingestion and inhalation are considered and specific filters for the physicochemical properties are applied [49]. The accuracy of the predictions obtained using the FAFDrugs 4 computational tool is about $70 \%$.

The physicochemical properties of small organic molecules may also be used for predicting their pharmacokinetics. The lipophilicity and polarity of a small molecule are useful molecular descriptors used to evaluate the passive human gastrointestinal absorption (GI) and the blood-brain barrier permeation (BBB), and they are considered when predicting the pharmacokinetics of phthalates using the SwissADME [51] and admetSAR [50] tools. SwissADME has been used to estimate the interactions of phthalates with cytochromes P450 (CYPs) that are known to be strongly involved in the metabolism of xenobiotics, namely CYP1A2, CYP2C9, CYP2C19, CYP2D6, and CYP3A4. This estimation is established using a support vector machine algorithm based on a large dataset of chemical compounds known to be substrates or inhibitors of the CYP enzymes. The estimation of the interactions of these chemicals with the CYPs enzymes informs about their pharmacokinetics associated xenobiotics-xenobiotics interactions. The SwissADME tool predicts pharmacokinetics profiles with an accuracy of $70 \%$. The AdmetSAR tool has been also used for estimating the pharmacokinetic profiles of the phthalates, the predictive accuracy of this tool being $76.7 \%$ [50].

Pred-Skin is a web-based tool that uses QSAR method for determining the skin sensitization potential: the binary predictions of human skin sensitization potentials are based on human data (the prediction accuracy is between $73 \%$ and $76 \%$ ), the binary and multiclass predictions of murine skin sensitization potentials are based on animal data (LLNA, the prediction accuracy is between $70 \%$ and $84 \%$ ), and the binary predictions based on non-animal data, i.e. Direct Peptide Reactivity Assay (DPRA), KeratinoSens, and the human Cell Line Activation Test (h-CLAT) with an accuracy of prediction between $80 \%$ and $86 \%[52,53]$. Usually, one positive result in one of the KeratinoSens ${ }^{\mathrm{TM}}$ or $\mathrm{h}$-CLAT tests designates a potential skin sensitizer compound, and two negative results point towards a non-sensitizer compound [66]. Within this study we only consider predictions based on human data, and on the KeratinoSens ${ }^{\mathrm{TM}}$ and h-CLAT tests. 
ENDOCRINE DISRUPTOME is a web facility based on the molecular docking approach using the AutoDock Vina algorithm, and predicts interactions between the chemical compound with 14 distinct human nuclear receptors: androgen receptor (AR), estrogen receptors $\alpha(E R \alpha)$ and $\beta(E R \beta)$, glucocorticoid receptor $(G R)$, liver $X$ receptors $\alpha(L X R \alpha)$ and $\beta(L R X \beta)$, peroxisome proliferator activated receptors $\alpha$ (PPRA $\alpha), \beta / \delta$ (PPRA $\beta)$, and $y$ (PPRA $y)$, retinoid $X$ receptor $\alpha(R X R \alpha)$ and thyroid receptors $\alpha(T R \alpha)$ and $\beta(\operatorname{TR} \beta)$. This tool predicts both agonistic and antagonistic (an) effects for the nuclear receptors $A R, E R \alpha, E R \beta$ and GR [54]. The predictions are quantitatively described by the sensitivity parameter (SE) based on the docking scores. Taking into account the values of this parameter, compounds are categorized in four classes: compounds with high probability of disrupting the nuclear receptors $(\mathrm{SE}<0.25)$; compounds with a medium probability of disrupting the nuclear receptors $(0.25<\mathrm{SE}<0.50)$; compounds with a low probability of disrupting the nuclear receptors $(0.50<\mathrm{SE}<0.75)$ and compounds predicted as non-affecting the nuclear receptors (SE>0.75). The accuracy of predictions made by this tool is about $72 \%$ [54].

Pred-hERG is another web facility based on the QSAR method that allows to obtain predictive models concerning the ability of a chemical compound to inhibit the human ether-à-go-go related gene (hERG) $\mathrm{K}^{+}$channels. This inhibition may conduct to cardiac side-effects such as heart arrhythmia and even possibly death [56]. The predictions made by the Pred-hERG tool have an accuracy of up to $89 \%$.

The Toxtree software has been used to obtain predictions concerning carcinogenicity and mutagenicity, which are two other important toxicological endpoints that must be assessed for the chemical compounds [57]. These predictions are based on applying the Crammer rules [67] for assessing the toxicity class, and the Benigni/Bossa rule for predicting the carcinogenicity and mutagenicity [68]. The accuracy of the Toxtree predictions is $70 \%$.

Molecular docking is used to assess the interactions of the phthalates with CYP1A2, CYP2C9, CYP2C19, CYP2D6 and CYP3A4, and is implemented under the SwissDock tool [58]. We have considered both blind and rigid docking. As the Protein Data Bank [69] contains numerous structures for the considered CYPs, the structural files with the following PDB entry codes are used in our molecular docking studies: 2HI4 for CYP1A2, 4NZ2 for CYP2C9, 4GQS for CYP2C19, 4XRZ for CYP2D6 and 4D6Z for CYP3A4. These structural files have been chosen so as to correspond to the complexes made by the enzymes with inhibitors and having the best resolution. The ligands, except HEM, have been removed from every spatial structure of the enzymes and the structures have been prepared for docking using the DockPrep utility within the Chimera software [70]. The Chimera software has been also used for obtaining the visualization and analysis results. 


\section{REFERENCES}

1. R.U. Halden, Annu Rev Public Health, 2010, 31, 179-194.

2. C. Gennings, R. Hausser, H.M. Koch, A. Kortenkamp, P.J. Lioy, P.E. Mirkes, B.A. Schwetz, Report to the U.S. Consumer Product Safety Commission by the Chronic hazard advisory panel on phthalates and phthalate alternatives, 2014. https://www.cpsc.gov/chap. Accessed 18 January 2019.

3. R.M. David, R.H. McKee, J.H. Butala, R.A. Barter, M. Kayser, Esters of aromatic mono-, di-, and tricarboxylic acids, aromatic diacids, and di-, tri-, or polyalcohols. In Patty's toxicology, E. Bingham, B. Cohrssen, C.H. Powell Eds, John Wiley \& Sons, New York, USA, 2001, Volume 6. pp. 635.

4. J. Doull, R. Cattley, C. Elcombe, B.G. Lake, J. Swenberg, C. Wilkinson, G. Williams, van M. Gemert, Regul Toxicol Pharmacol, 1999, 29, 327-357.

5. M. Wittassek, H.M. Koch, J. Angerer, T. Bruning, Mol Nutr Food Res, 2011, 55, 7-31.

6. W. Davidson-Urbain, P. Jouvet, M.P. Vélez, P. Ayotte, P. Monnier, Biomed J Sci \& Tech Res, 2018, 6, 5124-5129.

7. National Research Council. Intentional Human Dosing Studies for EPA Regulatory Purposes: Scientific and Ethical Issues, Committee on the Use of Third Party Toxicity Research with Human Research Participants Science, Technology, and Law Program, 2004, http://www.nap.edu/catalog/10927.html. Accessed 20 January 2019.

8. The National Institute for Occupational Safety and Health (NIOSH). https://www.cdc.gov/niosh/. Accessed between November 2017 - February 2019.

9. Occupational Safety and Health Administration (OSHA). https://www.osha.gov/. Accessed between November 2017 - June 2017.

10. Toxicology Data Network (TOXNET). https://toxnet.nlm.nih.gov/newtoxnet/hsdb.htm. Accessed between November 2017 - February 2019.

11. The PubChem database. https://pubchem.ncbi.nlm.nih.gov/\#. Accessed between November 2017 - June 2017.

12. BIBRA Toxicology Advice \& Consulting. https://www.bibrainformation.co.uk/toxicity-profiles-overview/. Accessed between November 2017 - February 2019.

13. D. Oral, P. Erkekoglu, B.K. Gumusel, M.-W. Chao, J Environ Pathol Toxicol Oncol, 2016, 35, 43-58.

14. R. Hauser, A.M. Calafat, Occup Environ Med, 2005, 62, 806-818.

15. M.H. Asghari, S. Saeidnia, M. Abdollahi, Int J Pharm, 2015, 11, 95-105.

16. I. Katsikantami, S. Sifakis, M.N. Tzatzarakis, E. Vakonaki, O.I. Kalantzi, A.M. Tsatsakis, A.K. Rizos, Environ Int, 2016, 97, 212-236. 
17. S.S.S. Rowdhwal, JX. Chen, Hindawi BioMed Research International, 2018, Article ID 1750368.

18. J. Knez, Reprod Biomed Online, 2013, 26, 440-448.

19. R. Rozati, P.P. Reddy, P. Reddanna, R. Mujtaba, Fertil Steril, 2002, 78, 11871194.

20. N. Pant, M. Shukla, D. Kumar Patel, Y. Shukla, N. Mathur, Y. Kumar Gupta, D.K. Saxena, Toxicol Appl Pharmacol, 2008, 231, 112-116.

21. R. Hauser, Int J Androl, 2008, 31,112-117.

22. L. López-Carrillo, R.U. Hernández-Ramírez, A.M. Calafat, L. Torres-Sánchez, M. Galván-Portillo, L.L. Needham, R. RuizRamos, M.E. Cebrián, Environ Health Perspect, 2010, 118, 539-544.

23. J.A. Hoppin, R. Ulmer, S.J. London, Environ Health Perspect, 2004, 112, 571574.

24. Y.-J. Lien, H.-Y. Ku, P.-H. Su, S.J. Chen, H.Y. Chen, P.C. Liao, W.J. Chen, S.L. Wang, Environ Health Perspect, 2015, 123, 95-100.

25. C.G. Bornehag, J. Sundell, C.J. Weschler, K.-G. Bornehag, J. Sundell, C.J. Weschler, T. Sigsgaard, B. Lundgren, M. Hasselgren, L. Hägerhed-Engman, Environ Health Perspect, 2004, 112, 1393-1397.

26. M. Matsumoto, M. Hirata-Koizumi, M. Ema, Regul Toxicol Pharmacol, 2008, 50, 37-49.

27. L.C. Li, S.L. Wang, Y.C. Chang, P.C. Huang, J.T. Cheng, P.H. Su, P.C. Liao, Chemosphere, 2011, 83, 1192-1199.

28. A. Araki, T. Mitsui, C. Miyashita, T. Nakajima, H. Naito, S. Ito, S. Sasaki, K. Cho, T. Ikeno, K. Nonomura, R. Kishi, PLoS One, 2014, 9, Article ID e109039.

29. H.-Y. Ku, P.-H. Su, H.-J. Wen, S.-L. Wang, PLoS One, 2015, 10, e0123309.

30. R. Rozati, P.P. Reddy, P. Reddanna, R. Mujtaba, Fertil Steril, 2002, 78, 11871194.

31. S.H. Swan, S. Sathyanarayana, E.S. Barrett, Hum Reprod, 2015, 30, 963-972.

32. S.H. Kim, S. Chun, J.Y. Jang, H.D. Chae, C.-H. Kim, B.M. Kang, Fertil Steril, 2011, 95, 357-359.

33. S.H. Kim, S. Cho, H.J. Ihm, Y.S. Oh, S.H. Heo, S. Chun, H. Im, H.D. Chae, C.H. Kim, B.M. Kang, J Clin Endocr Metab, 2015, 100, E1502-E1511.

34. B. Kolarik, K.Naydenov, M. Larsson, C.G. Bornehag, J. Sundell, Environ Health Perspect, 2008, 116, 98-103.

35. Y. Wang, S.H. Bryant, T. Cheng, J. Wang, A. Gindulyte, B.A. Shoemaker, P.A. Thiessen, S. He, J. Zhang, Nucleic Acids Res, 2017, 45, D955-D963.

36. P. Wolkoff, Int J Hyg Environ Health, 2013, 216, 371-394.

37. M. De Falco, M. Forte, V. Laforgia, Front Environ Sci, 2015, 3, 1-12.

38. M.A. Kamrin, J Toxicol Environ Health, Part B, 2009, 12, 157-174.

39. A.B. Raies, V.B. Bajic, Wiley Interdiscip Rev Comput Mol Sci, 2016, 6, 147-172.

40. C. Lu, C.M. Holbrook, L.M. Andres, Environ Health Perspect, 2010, 118, 125-130.

41. D. Craciun, D. Modra, A. Isvoran, AIP Conf Proc, 2015, 1694, 040007-1040007-6.

42. Y. Gao, T. An, Y. Ji, G. Li, C. Zhao, Environ Pollu, 2015, 206, 510-517. 
43. O.V. Tinkov, L.N. Ognichenko, V.E. Kuzmin, L.G. Gorb, A.P. Kosinskaya, N.N. Muratov, E.N. Muratov, F.C. Hill, J. Leszczynski, Struct Chem, 2016, 27, 191198.

44. I.A. Sheikh, R.F. Turki, A.M. Abuzenadah, G.A. Damanhouri, M.A. Beg, PLoS ONE, 2016, 11, Article ID e0151444.

45. A. Isvoran, A. Ciorsac, V. Ostafe, ADMET \& DMPK, 2017, 5, 192-200.

46. M. Roman, D.L. Roman, V. Ostafe, A. Ciorsac, Pharm Res, 2018, 35, Article ID 41.

47. M. Roman, D.L. Roman, V. Ostafe, A. Isvoran, J Bioinform Genomics Proteomics, 2018, 3, 1029.

48. V.M. Alves, E.N. Muratov, A. Zakharov, N.N. Muratov, C.H. Andrade, T. Tropsha, Food Chem Toxicol, 2018, 112, 526-534.

49. D. Lagorce, L. Bouslama, J. Becot, M.A. Miteva, B.O. Villoutreix, Bioinformatics, 2017, 33, 3658-3660.

50. F. Cheng, W. Li, Y. Zhou, J. Shen, Z. Wu, G. Liu, P.W. Lee, Y. Tang, J Chem Inf Model, 2012, 52, 3099-3015.

51. A. Daina, O. Michielin, V. Zoete, Sci Rep, 2017, 7, Article ID 42717.

52. R.C. Braga, V.M. Alves, E.N. Muratov, J. Strickland, N. Kleinstreuer, A. Trospsha, C.H. Andrade, Chem Inf Model, 2017, 57, 1013-1017.

53. V.M. Alves, S.J. Capuzzi, R.C. Braga, J.V.B. Borba, A.C. Silva, T. Luechtefeld, T. Hartung, C.H. Andrade, E.N. Muratov, A. Tropsha, ACS Sustainable Chem Eng, 2018, 6, 2845-2858.

54. K. Kolsek, J. Mavri, M. Sollner Dolenc, S. Gobec, S. Turk, J Chem Inf Model, 2014, 54, 1254-1264.

55. W. Gao, C.E. Bohl, J.T. Dalton, Chem Rev, 2005, 105, 3352-3370.

56. R.C. Braga, V.M. Alves, M.F. Silva, E. Muratov, D. Fourches, L.M. Lião, A. Tropsha, C.H. Andrade, Mol Inform, 2015, 34, 698-701.

57. G. Patlewicz, N. Jeliazkova, R.J. Safford, A.P. Worth, B. Aleksiev, SAR QSAR Environ Res, 2008, 19, 495-524.

58. A. Grosdidier, V. Zoete, O. Michielin, Nucleic Acids Res, 2011, 39, W270-W277.

59. S. Singh, S.S.-L. Li, Genomics, 2011, 97, 148-157.

60. J.J. Irwin, B.K. Shoichet, J Chem Inf Model, 2005, 45, 177-182.

61. C.A. Lipinski, F. Lombardo, B. Dominy, P.J. Feeney, Adv Drug Deliv Rev, 1997, 23, 3-25.

62. W.J. Egan, K.M. Merz, J.J. Baldwin, J Med Chem, 2000, 43, 3867-3877.

63. D.F. Veber, S.R. Johnson, H.Y. Cheng, B.R. Smith, K.W. Ward, K.D. Kopple, J Med Chem, 2002, 45, 2615-2623.

64. M.P. Gleeson, J Med Chem, 2008, 51, 817-834.

65. J.D. Hughes, J. Blagg, D.A. Price, S. Bailey, G.A. DeCrescenzo, R.V. Devraj, E. Ellsworth, Y.M. Fobian, M.E. Gibbs, R.W. Gilles, N. Greene, E. Huang, T. Krieger-Burke, J. Loesel, T. Wager, L. Whiteley, Y. Zhang, Bioorg Med Chem Lett, 2008, 18, 4872-4875.

66. Y. Otsubo, T. Nishijo, M. Miyazawa, K. Saito, H. Mizumachi, H. Sakaguchi, Reg Tox Pharm, 2017, 88,118-124.

67. G.M. Cramer, R.A. Ford, R.L. Hall, Food Cosmet Toxicol, 1978, 16, 255-276. 
68. R. Benigni, C. Bossa, Toxicol Mech Methods, 2008, 18, 137-147.

69. H.M. Berman, J. Westbrook, Z. Feng, G. Gilliland, T.N. Bhat, H. Weissig, I.N. Shindyalov, P.E. Bourne, Nucleic Acids Res, 2000, 28, 235-242.

70. E.F. Pettersen, T.D. Goddard, C.C. Huang, G.S. Couch, D.M. Greenblatt, E.C. Meng, T.E. Ferrin, J Comput Chem, 2004, 25, 1605-1612. 